\title{
Effect of vitamin D supplementation during pregnancy on maternal and neonatal outcomes: a systematic review and meta-analysis of randomized controlled trials
}

\author{
Faustino R. Pérez-López, M.D., Ph.D., ${ }^{a}$ Vinay Pasupuleti, M.D., Ph.D., ${ }^{b}$ Edward Mezones-Holguin, M.D.,, \\ Vicente A. Benites-Zapata, M.D.,, ${ }^{\text {P }}$ Priyaleela Thota, M.D., ${ }^{b}$ Abhishek Deshpande, M.D., Ph.D.e , \\ and Adrian V. Hernandez, M.D., Ph.D. d,f \\ a Department of Obstetrics and Gynecology, University of Zaragoza Faculty of Medicine and Lozano Blesa University \\ Hospital, Zaragoza, Spain; ${ }^{\mathrm{b}}$ Department of Medicine, Case Western Reserve University, Cleveland, Ohio; ${ }^{\mathrm{C}}$ Unit of \\ Analysis and Generation of Evidence in Public Health (UNAGESP), Instituto Nacional de Salud, Lima, Peru; ${ }^{d}$ School of \\ Medicine, Universidad Peruana de Ciencias Aplicadas (UPC), Lima, Peru; and e Medicine Institute Center for Value Based \\ Care Research and ${ }^{f}$ Health Outcomes and Clinical Epidemiology Section, Department of Quantitative Health Sciences, \\ Cleveland Clinic, Cleveland, Ohio
}

Objective: To assess the effects of vitamin D supplementation during pregnancy on obstetric outcomes and birth variables.

Design: Systematic review and meta-analysis of randomized controlled trials (RCTs).

Setting: Not applicable.

Patient(s): Pregnant women and neonates.

Intervention(s): PubMed and 5 other research databases were searched through March 2014 for RCTs evaluating vitamin D supplementation \pm calcium/vitamins/ferrous sulfate vs. a control (placebo or active) during pregnancy.

Main Outcome Measure(s): Measures were: circulating 25-hydroxyvitamin D [25(OH)D] levels, preeclampsia, gestational diabetes mellitus (GDM), small for gestational age (SGA), low birth weight, preterm birth, birth weight, birth length, cesarean section. Mantel-Haenszel fixed-effects models were used, owing to expected scarcity of outcomes. Effects were reported as relative risks and their 95\% confidence intervals (CIs).

Result(s): Thirteen RCTs ( $n=2,299)$ were selected. Circulating 25(OH)D levels were significantly higher at term, compared with the control group (mean difference: $66.5 \mathrm{nmol} / \mathrm{L}, 95 \%$ CI 66.2-66.7). Birth weight and birth length were significantly greater for neonates in the vitamin D group; mean difference: $107.6 \mathrm{~g}$ (95\% CI 59.9-155.3 g) and $0.3 \mathrm{~cm}$ (95\% CI 0.10-0.41 cm), respectively. Incidence of preeclampsia, GDM, SGA, low birth weight, preterm birth, and cesarean section were not influenced by vitamin D supplementation. Across RCTs, the doses and types of vitamin D supplements, gestational age at first administration, and outcomes were heterogeneous. Conclusion(s): Vitamin D supplementation during pregnancy was associated with increased circulating 25(OH)D levels, birth weight, and birth length, and was not associated with other maternal and neonatal outcomes. Larger, better-designed RCTs evaluating clinically relevant outcomes are necessary to reach a definitive conclusion. (Fertil Steril ${ }^{\circledR}$ 2015; $\square$ : $\square$. (C)2015 by American Society for Reproductive Medicine.)

Key Words: Vitamin D, pregnancy, maternal outcomes, neonatal outcomes, meta-analysis

Discuss: You can discuss this article with its authors and with other ASRM members at http:// fertstertforum.com/perezlopezf-vitamin-d-pregnancy/

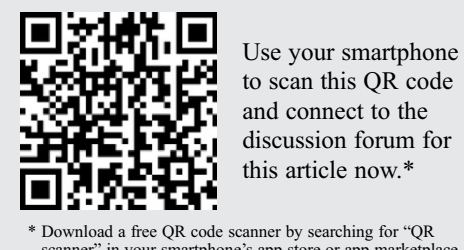

* Download a free $\mathrm{QR}$ code scanner by searching for " $\mathrm{QR}$
scanner" in your smartphone's app store or app marketplace.
Received October 19, 2014; revised February 11, 2015; accepted February 12, 2015.

F.R.P.-L. has nothing to disclose. V.P. has nothing to disclose. E.M.-H. has nothing to disclose. V.A.B.-Z has nothing to disclose. P.T. has nothing to disclose. A.D. has nothing to disclose. A.V.H. has nothing to disclose.

Reprint requests: Faustino R. Pérez-López, M.D., Ph.D., University of Zaragoza Faculty of Medicine, and Lozano Blesa University Hospital, Domingo Miral s/n, Zaragoza 50009, Spain (E-mail: faustino.perez@unizar.es).

Fertility and Sterility® Vol. $\square$, No. $\square, \mathbf{2} 2015$ 0015-0282/\$36.00

Copyright @2015 American Society for Reproductive Medicine, Published by Elsevier Inc.

http://dx.doi.org/10.1016/j.fertnstert.2015.02.019
V itamin $\mathrm{D}$ is an established fundamental nutritional factor responsible for regulation of bone metabolism, absorption of calcium and phosphate, and maintenance of muscle function. Observational studies suggest that vitamin $D$ is essential for many physiologic processes; 
thus, adequate levels are necessary and advantageous for optimal health (1-3). Despite its vitamin designation, cholecalciferol, or vitamin $\mathrm{D}_{3}$, can be synthesized by mammals from 7-dehydrocholesterol, via appropriate exposure to sunlight. Cholecalciferol and ergocalciferol (vitamin $\mathrm{D}_{2}$ ) can be obtained from the diet as well. In humans, both cholecalciferol and ergocalciferol are sequentially transformed into 25-hydroxyvitamin $\mathrm{D}_{3}$ [25(OH)D], 25-hydroxycholecalciferol, or calcidiol, in the liver, which are subsequently transformed in kidneys and other tissues into 1,25-dihydroxyvitamin $\mathrm{D}_{3}$ [1,25(OH)2D], 1,25dihydroxycholecalciferol or calcitriol (1-3).

Sufficient vitamin D concentrations are needed during pregnancy to address the increasing demand for calcium, by the fetus, during its growth and development $(3,4)$. Pregnant women who did not receive vitamin D supplementation showed a reduction in circulating 25(0H)D levels during the third trimester compared with the first trimester $(4,5)$. Several observational studies have reported that low maternal circulating 25(OH)D concentrations in pregnant women may have negative health consequences for both mothers and newborns $(3,6)$. Studies have suggested that lower levels of circulating 25(OH)D are associated with risks of recurrent pregnancy losses, preeclampsia, gestational diabetes, maternal infections, preterm birth, small-for-gestational-age (SGA) infants, and poor offspring health $(3,6,7)$.

Vitamin D supplementation may increase serum vitamin D levels in both mothers and infants $(8,9)$. However, what remains to be determined is whether vitamin D supplementation is protective against maternal morbid conditions, SGA, or intrauterine growth restriction, and whether it improves neonatal health. Findings from observational studies have been inconclusive, owing to the heterogeneity of the dose and duration of supplementation, the timing of supplementation initiation, maternal factors (overweight and obesity, general health before pregnancy), and measured endpoints.

The effects of vitamin D supplementation on maternal and neonatal outcomes in randomized controlled trials (RCTs) have been previously examined in 3 systematic reviews and/or meta-analyses $(7,9,10)$. These studies had several limitations, including deficiencies in study design (inclusion of quasi-randomized trials and observational studies), and are restricted by the outcomes evaluated. In addition, more RCTs have been published since the publication of the last meta-analysis. The current comprehensive systematic review and meta-analysis of RCTs includes studies published more recently. We evaluated current evidence on the effects of vitamin D supplementation during pregnancy on several maternal and neonatal endpoints. Clinical relevance of these findings and their potential translational clinical applications are discussed.

\section{MATERIALS AND METHODS}

This systematic review followed the Preferred Reporting Items for Systematic Reviews and Meta-Analyses (PRISMA) guidelines (11). Formal institutional review board approval was not required because this analysis pools published study data.

\section{Literature Search}

PubMed-Medline, EMBASE, Scopus, Web of Science, Cochrane Library, and www.clinicaltrials.gov were searched. A basic search strategy was developed for PubMed, and modified as needed for other databases; a search strategy was devised for each outcome (Supplemental Table 1, available online). The search time parameters were from inception of each database through March 2014. The language in which studies were written was not used to restrict selection, but only human studies were selected. References from the selected articles, including relevant review papers, were reviewed to identify all relevant studies.

\section{Inclusion and Exclusion Criteria}

The meta-analysis evaluated vitamin D supplementation, used alone and in combination with calcium and vitamin supplements, on pregnancy outcomes. Randomized controlled trials of pregnant women of any gestational or chronologic age and parity, without previous disease history, were included. Interventions of interest were: vitamin D alone vs. no treatment (placebo); vitamin D + calcium vs. no treatment (placebo); and vitamin D + calcium vs. calcium. Vitamins and ferrous sulfate were allowed in both trial arms. Controls of interest were: active controls, usual treatment without active control, and placebo.

In an RCT (8), all women received a standard prenatal multivitamin containing 400 international units (IU) of vitamin $\mathrm{D}_{3}$. In addition, all women received a vitamin $\mathrm{D}_{3}$ supplement of $0 \mathrm{IU}$ (placebo), 1,600 IU, or 3,600 IU of vitamin $\mathrm{D}_{3}$, for a total of $400 \mathrm{IU}, 2,000 \mathrm{IU}$, and 4,000 IU of vitamin D supplementation, respectively (Table 1). Exclusion criteria were: (1) no appropriate control group; (2) data were not available or could not be extracted for the study groups; and (3) multiple pregnancies.

\section{Prespecified Primary Outcomes}

Maternal primary outcomes were: preeclampsia (as defined by trialist); gestational diabetes (as defined by trialist); and vitamin D status at term (25-hydroxyvitamin D in nmol/L). Neonatal primary outcomes were: intrauterine growth restriction (as defined by trialist); low birth weight $(<2,500 \mathrm{~g})$; premature birth $(<37$ weeks of gestation); and birth weight in grams.

\section{Prespecified Secondary Outcomes}

Maternal secondary outcomes were: cesarean section and maternal mortality (death while pregnant or within 42 days of termination of pregnancy). Neonatal secondary outcomes were: birth length (cm); Apgar score of $<7$ at 5 minutes; stillbirth (as defined by trialist); neonatal infection (within 28 days after delivery); very low birth weight ( $<1,500 \mathrm{~g})$; early preterm birth ( $<34$ weeks gestation); and neonatal mortality (within 28 days after delivery).

\section{Study Selection and Data Extraction}

For this review, 2 sets of investigators independently reviewed titles and abstracts for eligibility. Disagreements regarding 


\section{TABLE 1}

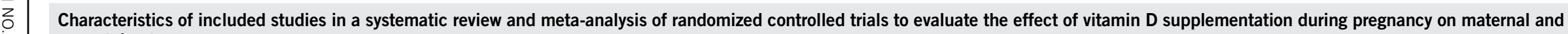
neonatal outcomes.

\section{First author, \\ year}

published

Asemi (14), 2013 Ira

Brooke (15), 1980 United

Kingdom

Delvin (16), 1986 France

Goldring (17)

2013

United

Private

Hashemipour (18), Iran 2014

\section{Hollis (8), $2011 \quad$ United University funds:}

$$
\text { States }
$$$$
\text { government }
$$

Hossain (19), 2014 Pakistan

Government

\section{Mallet (20), 1986 France}

Marya (21), 1987 India

Marya (22), 1988 India

NA

Roth (23), 2013 Bangladesh Private

$$
\text { foundation }
$$

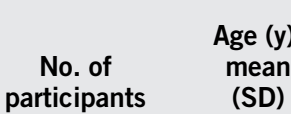

Age $(y)$,

(SD)

$\begin{array}{ll}27 & 24.8(3.6) \\ 27 & 25.3(4.2) \\ 67 & 23.7(3.1) \\ 59 & 23.9(4.8)\end{array}$

$\begin{gathered}\text { Treatment } \\ \text { group }\end{gathered}$
Placebo
$25(\mathrm{OH}) \mathrm{D}$
Placebo
Vitamin $\mathrm{D}_{2}$

$$
\text { NA No intervention }
$$

37.9 (36.9-39.9) No intervention 37.1 (36.5-38.8) Vitamin $\mathrm{D}_{2}$ $37.4(36.5-39.5)$ Vitamin $D_{3}$ $\begin{array}{ll}37.4(36.5-39.5) & \text { Vitamin } \mathrm{D}_{3} \\ 27.6(4.6) & \text { Elemental }\end{array}$

$$
\begin{array}{lll}
65 & 27.6(4.6) & \text { Elemental } \\
& \mathrm{Ca}+\mathrm{MVI}
\end{array}
$$$$
65 \quad 27.0(4.6)
$$

Elemental

$$
\mathrm{Ca}+\mathrm{MVI}+
$$

$166 \quad 27.0$ (5.6) Placebo

$$
167 \quad 27.4(5.7) \quad \begin{aligned}
& \text { MVI-400 } \\
& \text { MVI-400 }+
\end{aligned}
$$$$
\begin{array}{ll}
167 & 27.4(5.7) \quad M V l-400+ \\
\quad & \text { Vitamin } D_{3}
\end{array}
$$$$
16926.6(5.4) \quad \text { MVI-400 + }
$$

100

$25.2(4.4)$

$$
\text { Vitamin } D_{3}
$$

$\mathrm{FeSO}_{4}+$

calcium lactate

$$
26.0 \text { (3.1) } \quad \mathrm{FeSO}_{4}+\text { calcium }
$$

$$
\text { lactate }+
$$

25 (18-35) No intervention

$26(18-35) \quad$ Vitamin $D_{2}$

25 (19-36) Vitamin $D_{2}$

20-35 No intervention

24.1 (3.2) Ca + Vitamin D

$24.0(3.7) \quad$ Vitamin $D_{3}$

\section{$1,000 \mathrm{IU} / \mathrm{d}$}

200,000 IU single dose

$375 \mathrm{mg} / 1,200 \mathrm{IU} / \mathrm{d}$

600,000 IU, 2 doses

$\begin{array}{cc}\begin{array}{c}\text { Treatment } \\ \text { duration } \\ \text { (wk) }\end{array} & \begin{array}{c}\text { 25-OHD } \\ \text { quantification } \\ \text { method }\end{array} \\ 9 & \text { ELISA } \\ 8-12 & \begin{array}{c}\text { Protein-binding } \\ \text { assay } \\ 12\end{array} \\ 13 & \begin{array}{c}\text { Radioligand } \\ \text { assay }\end{array} \\ 8 & \text { ELISA } \\ 24-28 & \text { RIA }\end{array}$

\section{Gestational}

age at

sampling

(wk)

Primary endpoint

25 C-reactive protein

28-32 Maternal and infant calcium homeostasis,

fetal growth
26-27 Maternal and neonatal

27 Wheeze prevalence

24-26 Newborn length at birth

24-28 RIA

12-16 Maternal and neonatal 25-OHD at delivery

$$
3,600
$$

200/600 mg/d

4,000
20 Chemiluminescence immunoassay
20 Neonatal Vitamin D status, obstetric outcomes, neonatal growth and Apgar scores
12 Protein-binding NA Maternal and cord blood 25(OH)D $1,25(\mathrm{OH}) 2 \mathrm{D}$ 16-20 NA

12 NA

NA Blood pressure

NA Maternal and cord sera biochemical parameters, fetal birth weight. and anthropometry

$\begin{array}{ccc}\text { High-performance } & 27.9 & \text { Maternal and neonatal } \\ \text { liquid } & \text { (mean) } & \text { 25(OH)D, maternal }\end{array}$ chromatography serum calcium 


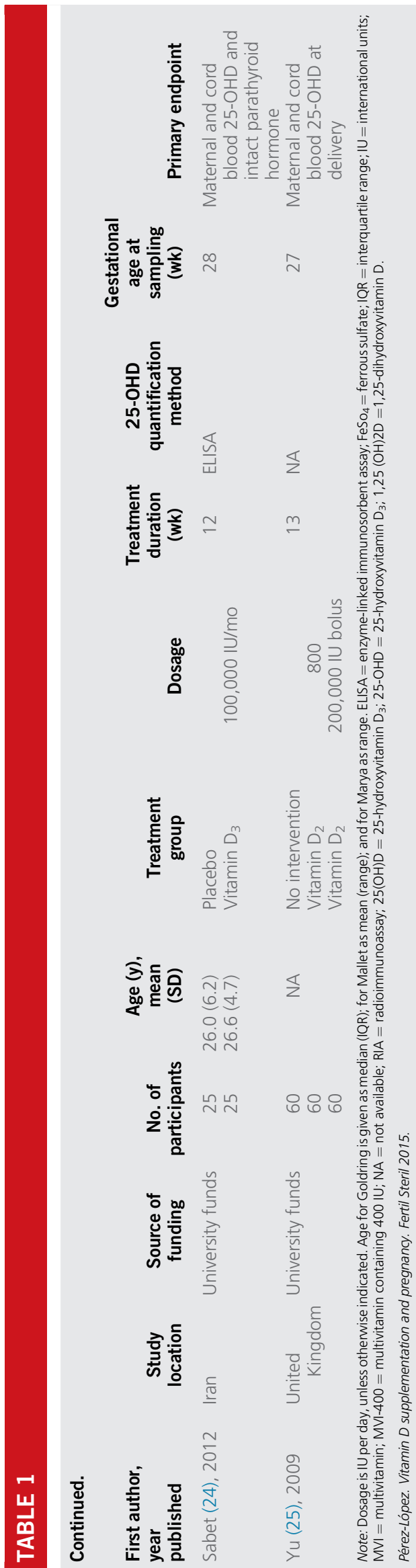

\section{FIGURE 1}

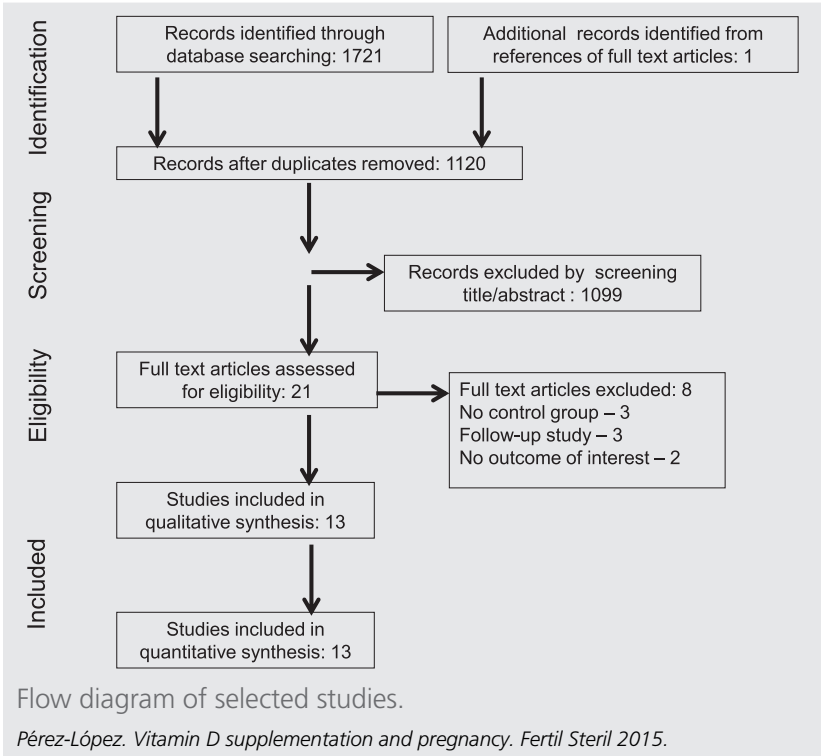

abstract selection were resolved by consensus or discussion with another investigator not in the first 2 groups. The study selection flow chart was created according to PRISMA guidelines (11).

The 2 sets of investigators independently extracted relevant data (participants, specific vitamin D intervention, and outcome characteristics) from each full-text article and recorded the data directly onto previously designed dataextraction spreadsheets. Entries were compared for accuracy, and any discrepancies were resolved by consensus or discussion with another investigator not in the first 2 groups, if needed.

Authors of original studies were contacted if necessary for unpublished information relevant to the study. In cases of duplicate publications or multiple reports of the primary study, data extraction was optimized by using the best information available for all items from the same study. The longest follow-up period associated with primary or secondary outcomes was used.

\section{Risk of Bias Assessment in Randomized Controlled Trials}

Two sets of investigators independently evaluated the risk of bias from each eligible RCT. Any discrepancies were resolved by consensus or discussion with another investigator. The Cochrane Collaboration tool for assessing risk of bias in RCTs was used (12). The following items were evaluated: generation of the allocation sequence (selection bias); concealment of the allocation sequence (selection bias); blinding (detection and performance bias); blinding of participants and personnel to outcome assessment; incomplete outcome data (attrition bias); selective outcome reporting (reporting bias); and other biases. For each RCT, each item was described as having either a low risk of bias, a high risk of bias, or an unclear risk of bias. 


\section{TABLE 2}

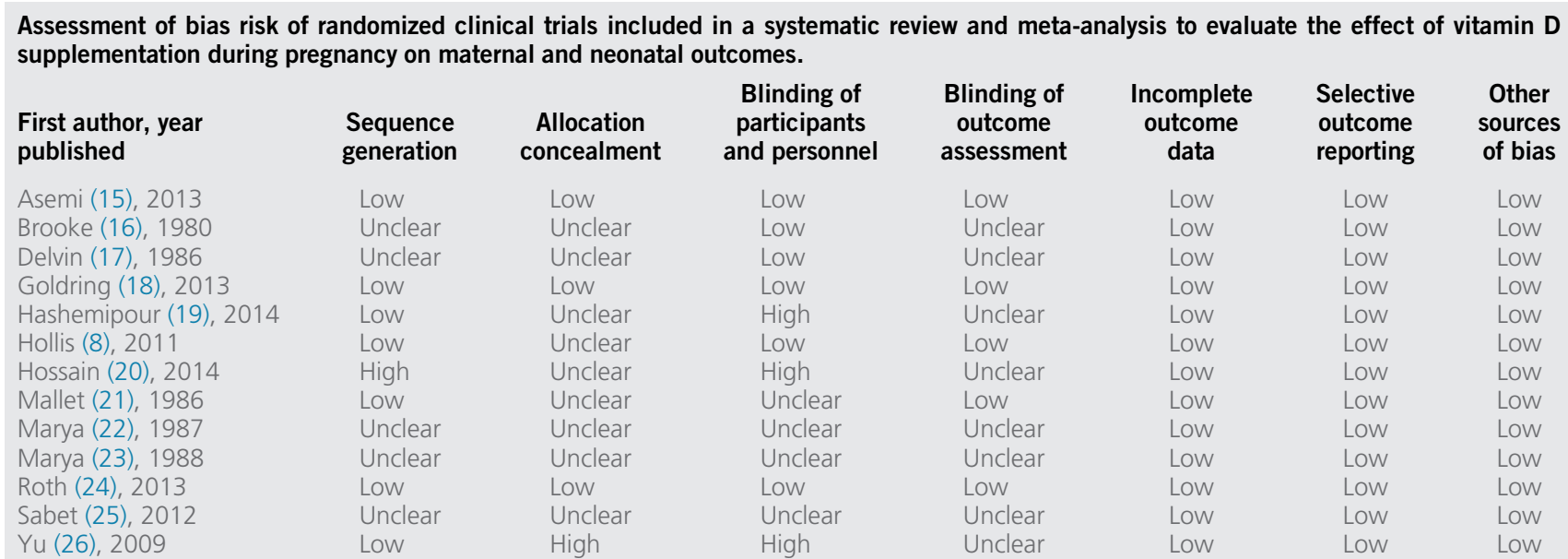

Note: Bias risk was determined according to the Cochrane risk of bias tool (12), using the following questions by category. Sequence generation: Was the allocation sequence adequately generated? Allocation concealment: Was allocation adequately concealed? Blinding of participants, personnel and outcome assessors: Was knowledge of the allocated intervention adequately prevented during the study? Incomplete outcome data and withdrawals: Were intention-to-treat analyses performed? Had participants withdrawn from the study? Selective outcome reporting: Free of selective reporting? Other sources of bias: Was sample size calculated? Were inclusion and exclusion criteria and baseline characteristics defined? Were conflicts of interest reported?

Pérez-López. Vitamin D supplementation and pregnancy. Fertil Steril 2015.

\section{Statistical Analysis}

For analyses, fixed-effects models and the Mantel-Haenszel method were used, owing to an anticipated scarcity of events $(<10 \%$ of the total number of individuals in an arm). This method has better statistical properties than the inverse variance method when events are few; in particular, estimates of the standard errors of the effect estimates that are used with the this method may be poor (13). Outcomes data available in $\geq 3$ studies were meta-analyzed.

Associations were reported as relative risks (RRs) and their 95\% confidence intervals (CIs). Heterogeneity was tested with the Cochrane $\chi^{2}$ test, and the degree of heterogeneity was quantified with the $I^{2}$ statistic and its 95\% CI. An $I^{2}$ value between 30\% and 60\% was described as moderate heterogeneity. Publication bias was assessed with the funnel plots and formally tested with the Egger's test. If enough information was available, we planned to perform subgroup analyses by level of vitamin $D$ at the beginning of pregnancy (normal vs. abnormal), and by Cochrane's risk-of-bias level. If vitamin D levels were provided in $\mathrm{ng} / \mathrm{mL}$, values were converted using the formula: $1 \mathrm{ng} / \mathrm{mL}=2.5 \mathrm{nmol} / \mathrm{L}$. All analyses were done with RevMan 5.1 (14) and the package metafor of R 3.0.1 (www.r-project.org).

\section{RESULTS}

\section{General Characteristics of Studies}

After the evaluation of 1,120 abstracts from primary and secondary sources, 1,099 were excluded; 21 were assessed from the full text. From these, 8 studies were excluded (Fig. 1). Thus, 13 RCTs $(\mathrm{n}=2,299)$ were included in this systematic review. All were published between 1980 and 2014, were conducted in both developing and developed countries, and had sample sizes in the range 40-400 pregnant women, most age $<30$ years $(8,15-26)$ (Table 1$)$. From the total of 13
RCTs, 3-8 were included in the various meta-analyses, depending on the health outcome studied.

Vitamin $\mathrm{D}_{2}$ or $\mathrm{D}_{3}$, alone or in combination with multivitamins, calcium, or iron were the interventions; the controls were active, placebo, or no intervention. In 1 RCT (8), all women received a standard prenatal multivitamin containing $400 \mathrm{IU}$ of vitamin $\mathrm{D}_{3}$, and an additional vitamin $\mathrm{D}_{3}$ supplement of either $0 \mathrm{IU}$ (placebo), 1,600 IU, or 3,600 IU, for totals of $400 \mathrm{IU}, 2,000 \mathrm{IU}$, and 4,000 IU of vitamin D supplementation, respectively (Table 1). The duration of the vitamin D intervention was 8-28 weeks of gestation.

Outcomes were heterogeneous, and the most frequently reported outcomes were maternal and neonatal 25(OH)D levels at delivery. Vitamin D levels were measured mainly by using competitive binding assays: an enzyme-linked immunosorbent assay in 3 RCTs, protein-binding assays in 2, a radioimmunoassay in 2, a radioligand assay in 1 , and a chemiluminescence assay in 1 , Measures were either not stated or not available in 3 RCTs. In 1 study, measurement was done using high-performance liquid chromatography (Table 1).

\section{Risk-of-Bias Assessment}

Risk-of-bias assessment is shown in Table 2. Of the 13 included studies, 3 had a low risk of bias, 2 had a high risk of bias, and the remaining 8 had an unclear risk of bias.

\section{Meta-Analyses of Primary Outcomes}

As expected, the 25(OH)D levels at delivery were higher in women who received the intervention vs. those in the control group (mean difference: $66.5 \mathrm{nmol} / \mathrm{L}$, 95\% CI 66.2-66.7) (Fig. 2A). Incidences of preeclampsia and gestational diabetes were similar in women with vs. without the vitamin D intervention (RR 0.88, 95\% CI 0.51-1.52; and RR 1.05, 95\% CI 0.60-1.84, respectively) (Fig. 2B and 2C). 


\section{FIGURE 2}

\begin{tabular}{|c|c|c|c|c|c|c|c|}
\hline \multirow[b]{2}{*}{ Stucty or Subgroup } & \multicolumn{3}{|c|}{ Vitamin D } & \multicolumn{3}{|c|}{ Control } & \multirow[b]{2}{*}{ Weight } \\
\hline & Mean & SD & Total & Mean & SD & Total & \\
\hline Asemi 2013 & 9.25 & 1.07 & 27 & -3 & 0.78 & 27 & $23.7 \%$ \\
\hline Brooke 1980 & 147.8 & 1.65 & 59 & -3.8 & 0.45 & 67 & $31.3 \%$ \\
\hline Delvin 1986 & 40 & 3.95 & 20 & 7.5 & 4.51 & 20 & $0.9 \%$ \\
\hline Hashemipour 2014 & 80 & 3.86 & 65 & -4 & 2.53 & 65 & $4.7 \%$ \\
\hline Hollis 2011 high & 52.8 & 3.53 & 169 & 17.3 & 3.53 & 166 & $10.3 \%$ \\
\hline Hollis 2011 low & 40 & 3.16 & 167 & 17.3 & 3.53 & 166 & $11.4 \%$ \\
\hline Roth 2013 & 89 & 4 & 80 & -5.6 & 3.09 & 80 & $4.8 \%$ \\
\hline Sabet 2012 & 69.88 & 18.43 & 25 & -22.5 & 14.09 & 25 & $0.1 \%$ \\
\hline Yu 2009 high & 7.5 & 2.45 & 60 & 2.75 & 1.99 & 60 & $9.3 \%$ \\
\hline Yu 2009 low & 20.5 & 4.6 & 60 & 2.75 & 1.99 & 60 & $3.7 \%$ \\
\hline Total $(95 \% \mathrm{Cl})$ & & & 732 & & & 736 & $100.0 \%$ \\
\hline \multicolumn{8}{|c|}{ Heterogeneity: $\mathrm{Chi}^{2}=246023.48, \mathrm{df}=9(\mathrm{P}<0.00001) ; \mathrm{I}^{2}=100 \%$} \\
\hline
\end{tabular}

B

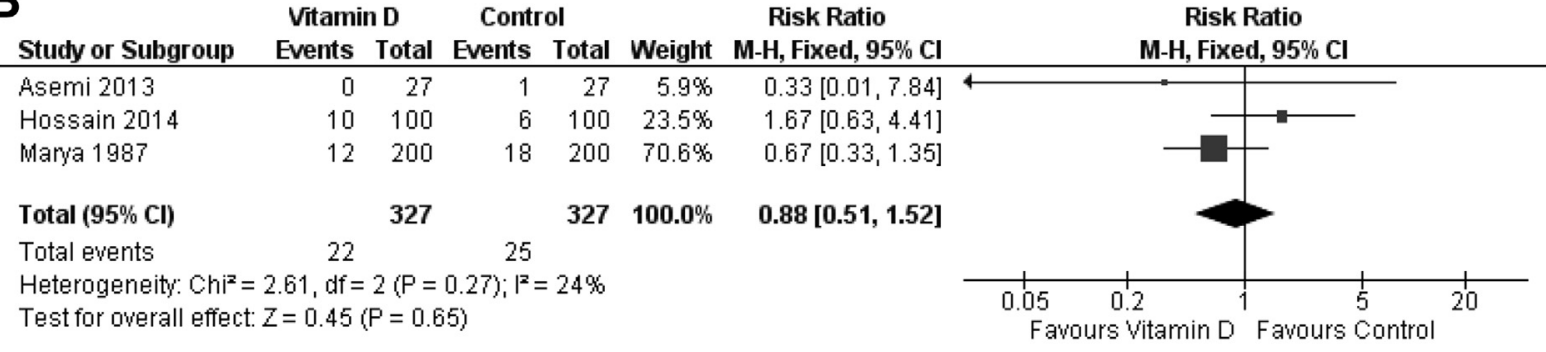

C

Study or Subgroup

Asemi 2013

Hashemipour 2014

Hossain 2014

Vitamin D

Control

Risk Ratio

Risk Ratio

Events Total Events Total Weight $\mathrm{M}-\mathrm{H}$, Fixed, 95\% Cl

$\begin{array}{rr}0 & 27 \\ 1 & 65 \\ 19 & 100\end{array}$

$\begin{array}{lll}1 & 27 & 7.5 \%\end{array}$

$\begin{array}{lll}0 & 65 & 2.5 \%\end{array}$

$18 \quad 100 \quad 90.0 \%$

$0.33[0.01,7.84]$

$3.00[0.12,72.31]$

Total $(95 \% \mathrm{Cl})$

192

$192100.0 \%$

$1.06[0.59,1.89]$

Total events 20 19

Heterogeneity: $\mathrm{Chi}^{2}=0.93, \mathrm{df}=2(\mathrm{P}=0.63) ; \mathrm{I}^{2}=0 \%$

Test for overall effect: $Z=0.17(P=0.86)$

$1.05[0.60,1.84]$

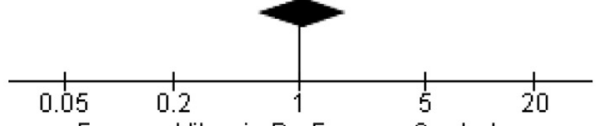

Meta-analyses of the effect of vitamin D intervention on primary outcomes (A) circulating 25-OHD; (B) preeclampsia; (C) gestational diabetes; (D) small for gestational age; (E) low birth weight; (F) preterm birth; (G) birth weight, and secondary outcomes (H) birth length; and (I) cesarean section. $\mathrm{Cl}=$ confidence interval; D2 = supplement of vitamin D2; D3 = supplement of vitamin D3; df = degrees of freedom; high = higher dose; low = lower dose; $\mathrm{M}-\mathrm{H}=$ Mantel-Haenszel; 25(OH)D = 25-hydroxyvitamin $\mathrm{D}_{3}$.

Pérez-López. Vitamin D supplementation and pregnancy. Fertil Steril 2015.

In contrast with the control group, incidences of SGA (RR $0.78,95 \%$ CI 0.50-1.21), low birth weight (RR 0.72, 95\% CI $0.44-1.16)$, and preterm birth (RR 1.26, 95\% CI 0.60-2.63) in neonates were not different for the vitamin D intervention groups (Fig. 2D-2F). Birth weight was slightly but significantly greater for the vitamin $\mathrm{D}$ groups (mean difference: $108 \mathrm{~g}, 95 \%$ CI 60-155 g) (Fig. 2G).

\section{Meta-Analyses of Secondary Outcomes}

Neonatal birth length was slightly but significantly greater in the vitamin D intervention group (mean difference: $0.3 \mathrm{~cm}$, 95\% CI 0.19-0.41) (Fig. 2H). The incidence of cesarean section was not different between groups (RR 0.94, 95\% CI 0.78-1.13) (Fig. 2I). Other prespecified secondary outcomes were not reported in $\geq 3$ studies and thus were not meta-analyzed. The prespecified subgroup analyses were not conducted, owing to the scarcity of studies providing relevant information.

\section{DISCUSSION}

This systematic review and meta-analysis of RCTs showed a significant increase in circulating $25(\mathrm{OH}) \mathrm{D}$ in pregnant women who received vitamin D supplementation. Birth weight and birth length were slightly but significantly greater in the neonates of mothers who received vitamin D supplements, compared with those who did not. In addition, the current investigation found that the incidence of preeclampsia, gestational diabetes, preterm birth, SGA, and cesarean section were similar among pregnant women who did, vs. did not, receive vitamin D supplementation. 


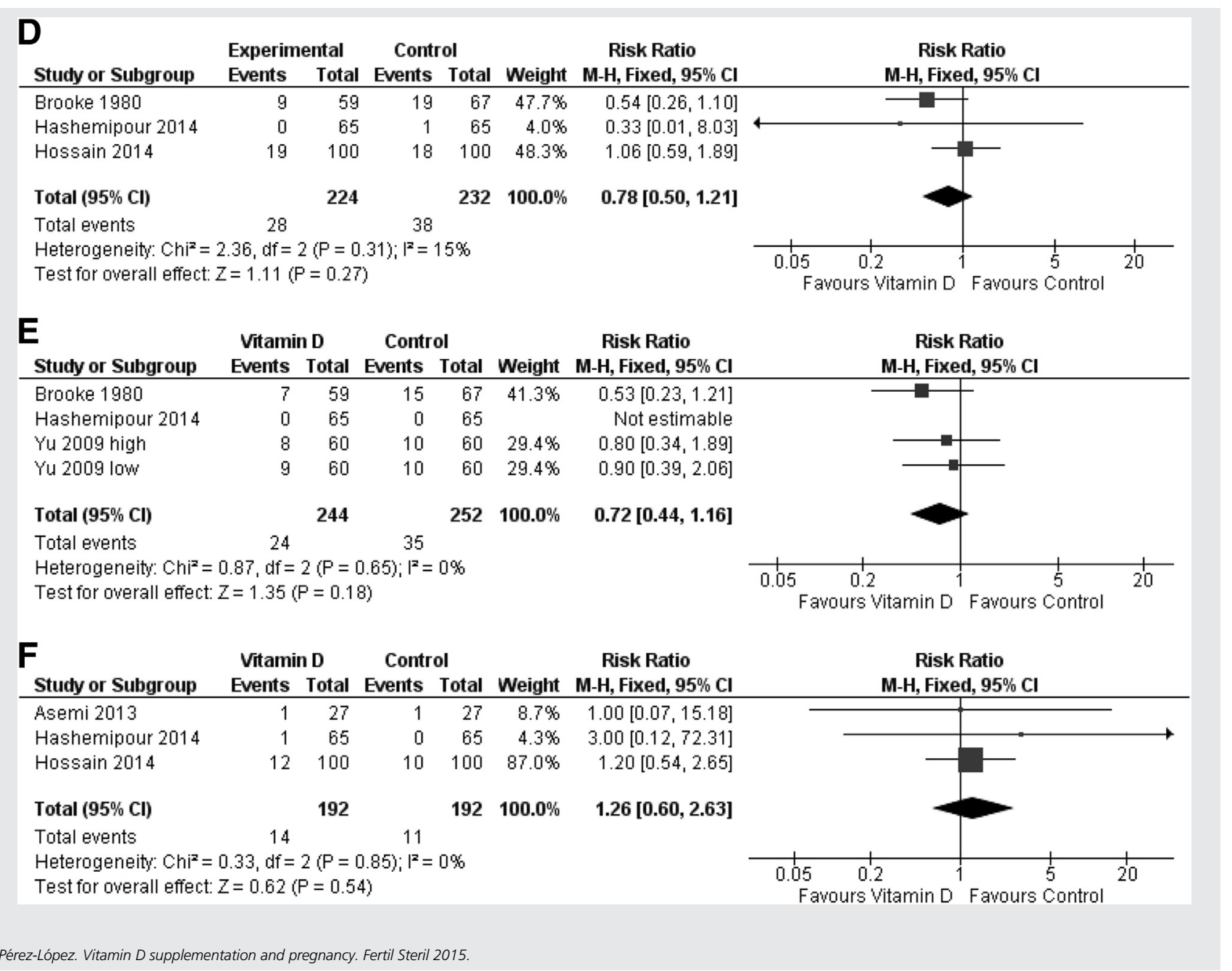

\section{Vitamin D Supplementation and Fetal Birth Weight and Length}

Although the improvements in birth weight (found in 8 RCTs) and length (found in 6 RCTs) were rather small, they suggest indirectly that vitamin $\mathrm{D}$ supplementation exerts a positive effect on fetal cell mass and function, skeletal mineralization, and metabolism (27). On the other hand, small differences in gestational age could be contributing to the effect (28). The small effects on birth size seem less likely to be a function of growth restriction (both the SGA estimate and the low birth weight point estimates are lower, but not significantly, for those in the vitamin D supplementation group) than of the gestational age distribution, because the preterm estimate is slightly elevated, though again, not significantly so.

In any case, fetal growth is a complex process dependent on many factors, including genetic background, birth interval, trophoblast implantation, placental development, nutrition, and physical activity (29-31). Thus, vitamin D may play a minor role in fetal growth, compared with other factors. Future research should be based on more- standardized growth-assessment procedures (32), to control for some of the limitations found in the available evidence.

\section{Vitamin D Supplementation and Low Fetal Birth Weight, Small for Gestational Age, and Preterm Birth}

An inverse correlation of cord blood 25(OH)D levels at delivery and birth weight has been reported (8), whereas other studies have shown that newborns of mothers with severe vitamin D deficiency had shorter birth length, and smaller head circumference and birth weight (33). In the current meta-analysis, including 4 RCTs, vitamin D supplementation did not prevent the risk of low birth weight $(<2,500 \mathrm{~g})$. Pooled data from 3 RCTs in this study showed no significant effect of vitamin D supplementation on the risk of SGA.

Reports are conflicting on the role of vitamin D and the risk of preterm birth. Premature amniotic membrane rupture and preterm delivery have been associated with vitamin D deficiency and inflammatory response $(34,35)$, although 
FIGURE 2 Continued

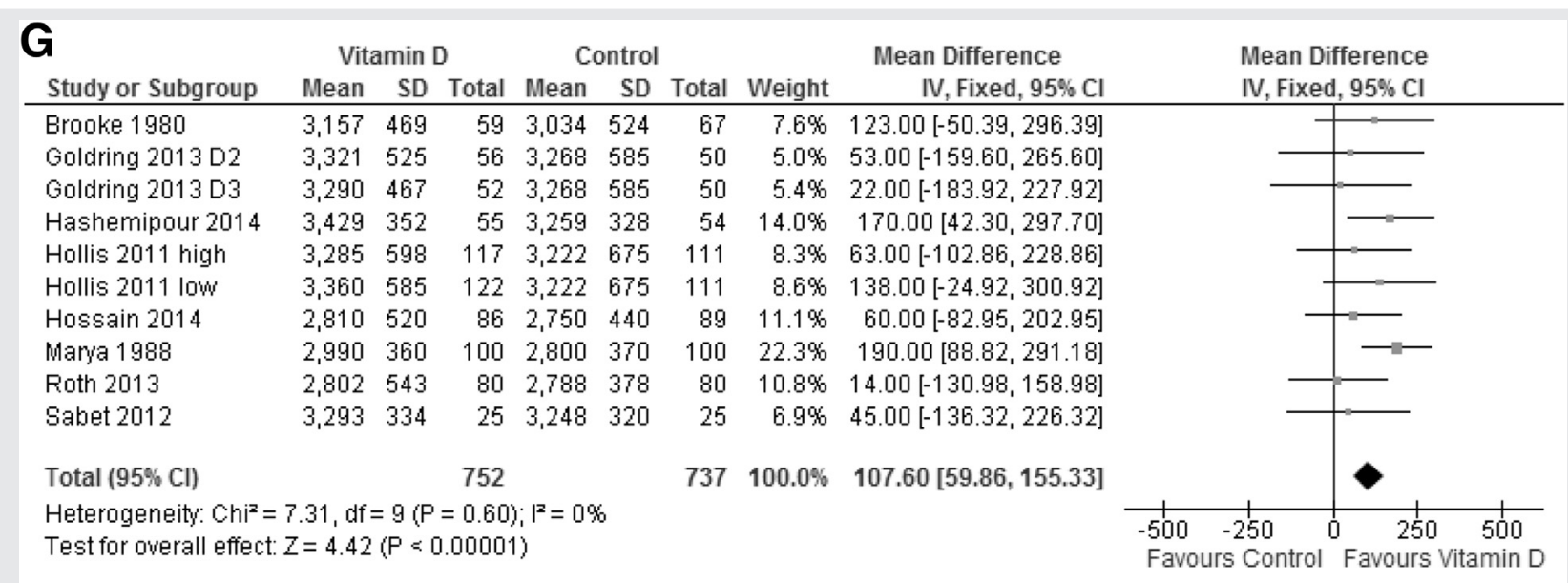

\section{H}

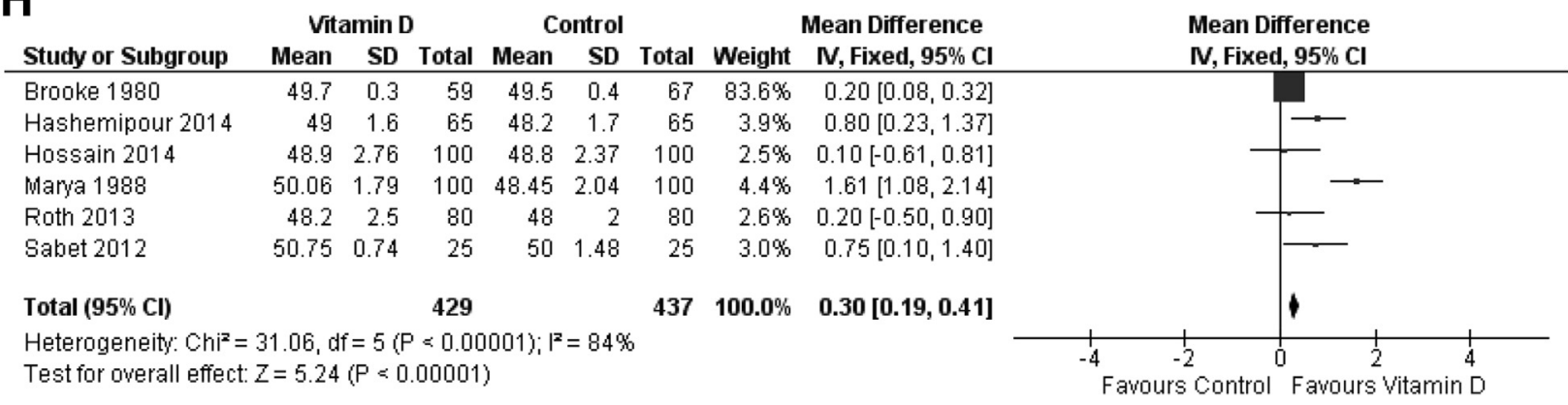

1

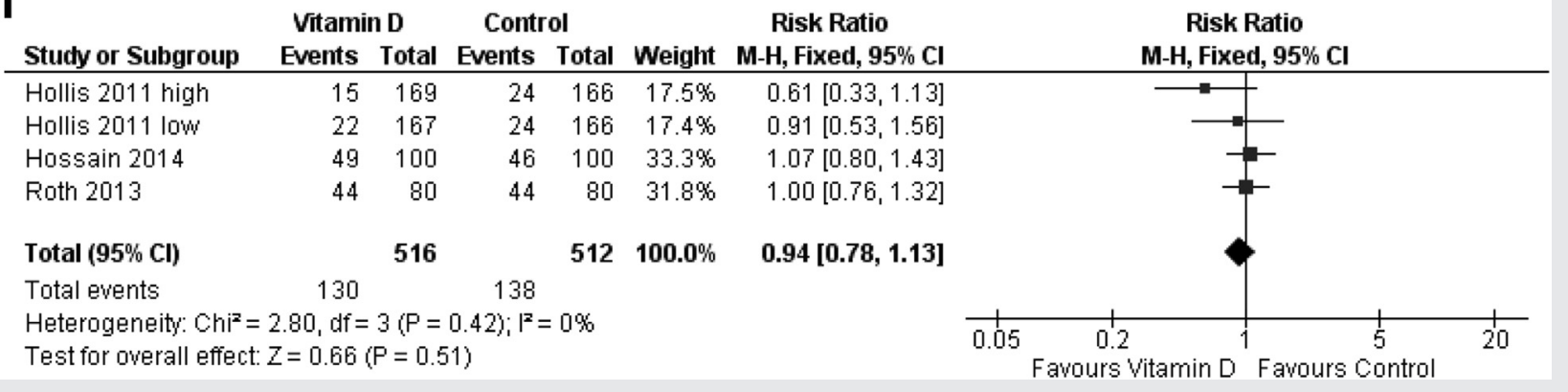

Pérez-López. Vitamin D supplementation and pregnancy. Fertil Steril 2015.

other studies did not confirm an association between 25(OH)D status and preterm birth $(36,37)$. Pooled analysis of 4 RCTs in the current study showed no significant effect of vitamin D supplementation on prevention of preterm birth. Some recent publications have reported alterations in the cervicovaginal fluid content of vitamin $\mathrm{D}$ and vitamin $\mathrm{D}$ binding protein (VDBP) as biomarkers of vaginal inflammation and preterm birth risk several weeks before delivery (38). Future research on vitamin D supplementation and preterm birth should include the study of cervicovaginal VDBP and serum 1,25(OH)2D, along with 25(OH)D, to monitor the response to various doses of vitamin D supplements.

\section{Vitamin D Supplementation and Preeclampsia}

Controversy remains regarding the clinical and epidemiologic evidence on the relationship between low maternal vitamin D levels and the risk of preeclampsia $(39,40)$. In the current meta-analysis, 3 RCTs assessed vitamin D supplementation and preeclampsia risk and did not find any significant association between them. However, placenta dysfunction has a major pathogenetic role on preeclampsia development, and the increased oxidative stress produces various vitamin D-related alterations (expression of VDBP, 25-hydroxylase, 1a-hydroxylase, and vitamin D receptor) (41). On the other hand, 1 RCT (20) included in our 
meta-analysis used a combination of vitamin $\mathrm{D}$, ferrous sulfate (200 mg), given twice daily, and $600 \mathrm{mg}$ of calcium lactate daily for the treatment group, vs. ferrous sulfate and calcium in the control group. The other two studies $(15,22)$ used vitamin $\mathrm{D}$ in the treatment group, vs. placebo. The results from the Hossain et al. study (20) study seem to have driven the overall RR toward the null. Some studies suggest that calcium supplementation reduces preeclampsia risk $(42,43)$.

\section{Vitamin D Supplementation and Gestational Diabetes Mellitus}

Low vitamin D levels have been associated with altered glucose homeostasis in both in vitro and observational studies (44-46). In the current meta-analysis of 3 RCTs that included a small number of women overall, no significant benefit of vitamin D supplementation was found in relation to risk of gestational diabetes. However, several confounding factors have not been controlled for in the available data. Excessive weight gain during pregnancy contributes to both the risk of gestational diabetes and low maternal vitamin D levels, as this vitamin is fat soluble and migrates from the blood to fat tissue (47). In addition, higher levels of VDBP in obese patients are associated with a reduced fraction of the bioactive, unbound 15(OH)D (48).

\section{Vitamin D Supplementation and Cesarean Section}

Low circulating blood 25(OH)D levels have been reported to be associated with an increased rate of cesarean section $(49,50)$. On the contrary, first-trimester maternal vitamin D levels were similar in women who subsequently have a vaginal delivery vs. those who deliver by elective or emergency cesarean section (51). In the current study, 4 RCTs were pooled; no significant effect was found of vitamin D supplementation on cesarean section rates. Cesarean section indications are quite variable, owing to many factors relating to the obstetric experience, hospital facilities, and other issues that are very difficult to control.

\section{Limitations of Previous Meta-Analyses}

De Regil et al. (9), in an evaluation of RCTs and quasirandomized trials, found that vitamin D supplementation was not associated with preeclampsia or low birth weight. Thorne-Lyman and Fawzi (7) evaluated observational studies and RCTs and found an association of vitamin D supplementation and diet, with a lower incidence of low birth weight; other outcomes were not significantly affected by the interventions. Finally, Harvey et al. (10) assessed observational studies and RCTs for the effect of serum vitamin D levels, or supplementation of women with vitamin D or food containing vitamin $\mathrm{D}$ on maternal and neonatal outcomes. In comparison with these 3 earlier systematic reviews (Supplemental Table 2, available online), the current metaanalysis predefined several other relevant maternal and neonatal outcomes, excluded observational studies that were prone to several biases, and included a larger number of RCTs and time spans ranging to as recently as 2014 .

\section{Limitations of Current Meta-Analyses}

This systematic review has several limitations. The available studies were heterogeneous in terms of dose, type, and duration of vitamin D supplementation, as well as maternal and neonatal endpoints. In addition, serum 25(OH)D levels were quantified using various types of competitive binding assays, with only 1 RCT using high-performance liquid chromatography, the gold standard of measurement. Competitive binding assays were the most-often used method of assessing $25(\mathrm{OH}) \mathrm{D}$. These methods, compared with high-performance liquid chromatography, underestimate serum levels, owing to differences in affinity between the antibodies or binding elements used $(52,53)$.

Methodologic deficiencies are an additional limitation; a few RCTs initiated supplementation in the second half of pregnancy, and many clinical conditions (e.g., preeclampsia and gestational diabetes) cannot be prevented as the several biochemical, metabolic, and vascular changes have already been made. In addition, definitions of some clinical outcomes varied among included studies. Given that many studies were carried out in developing countries, the possibility of maternal and child undernutrition in the studied population cannot be ruled out (54). In such cases, the small contribution of vitamin D supplementation would not be potent enough to neutralize the basal nutritional status.

Other confounding factors not assessed in available RCTs include diet, vitamin D content, seasonality, body weight gain during pregnancy, ethnicity, and skin characteristics $(3,4,6$, 39 , 53, 55). Birth spacing was not reported in the RCTs, and therefore we could not control for this factor. Most of the included studies had an unclear risk of bias, according to the Cochrane Risk of Bias Tool.

Recognizing the limitations of studies included in metaanalyses may stimulate future studies with better design and methods that will improve available evidence and definitively define the role of vitamin D in pregnancy and neonatal health. Future RCTs should consider initiating vitamin D supplementation early in pregnancy or even before pregnancy, controlling for confounding factors, using higher and moresustained doses than those studied so far, with moreobjective endpoints (e.g., ultrasound assessment and serial measurement of biochemical markers) (31, 54-56).

Finally, a remaining issue is whether a linear correlation exists between vitamin D supplementation and maternal and neonatal outcomes, and whether various obstetric endpoints have varying cutoffs. Some experimental and clinical studies suggest that hypervitaminosis D may have negative effects on obstetrics endpoints $(57,58)$. Thus, the possibility that some outcomes could have U-shaped associations, with risks at both low and high levels, cannot be disregarded (59). However, vitamin D supplementation of up to 4,000 IU per day seemed to be safe during pregnancy $(8,20)$.

The current state of the evidence is controversial for some other endpoints, and the actual benefit of vitamin D supplementation in pregnancy remains unclear. Additional longitudinal studies may clarify the actual impact of vitamin D deficiency during pregnancy. Randomized trials are required to define the benefits of vitamin D supplementation 
in reducing the incidence of adverse outcomes in mothers and infants. Fulfilling at least the recommended dietary allowance of 600 IU per day seems reasonable (60-62), until more-robust evidence is available that higher daily doses of vitamin D are beneficial. Even this minimal amount of vitamin D (600 IU per day) is not received by many pregnant women worldwide $(63,64)$.

\section{REFERENCES}

1. Pérez-López FR, Chedraui P, Fernández-Alonso AM. Vitamin D and aging: beyond calcium and bone metabolism. Maturitas 2011;69:27-36.

2. Wacker M, Holick MF. Vitamin D-effects on skeletal and extraskeletal health and the need for supplementation. Nutrients 2013;5:111-48.

3. Ponsonby AL, Lucas RM, Lewis S, Halliday J. Vitamin D status during pregnancy and aspects of offspring health. Nutrients 2010;2:389-407.

4. Fernández-Alonso AM, Dionis-Sánchez EC, Chedraui P, GonzálezSalmerón MD, Pérez-López FR. Spanish Vitamin D and Women's Health Research Group. First-trimester maternal serum 25-hydroxyvitamin D status and pregnancy outcome. Int J Gynecol Obstet 2012;116:6-9.

5. Hossain N, Khanani R, Hussain-Kanani F, Shah T, Arif S, Pal L. High prevalence of vitamin D deficiency in Pakistani mothers and their newborns. Int J Gynecol Obstet 2011;112:229-33.

6. Pérez-López FR. Vitamin D: the secosteroid hormone and human reproduction. Gynecol Endocrinol 2007;23:13-24.

7. Thorne-Lyman A, Fawzi WW. Vitamin D during pregnancy and maternal, neonatal and infant health outcomes: a systematic review and meta-analysis. Pediatr Perinat Epidemiol 2012;26(Suppl 1):75-90.

8. Hollis BW, Johnson D, Hulsey TC, Ebeling M, Wagner CL. Vitamin D supplementation during pregnancy: double-blind, randomized clinical trial of safety and effectiveness. J Bone Miner Res 2011;26:2341-57.

9. De Regil LM, Palacios C, Ansary A, Kulier R, Peña-Rosas JP. Vitamin D supplementation for women during pregnancy. Cochrane Database Syst Rev 2012; 2:CD008873.

10. Harvey NC, Holroyd C, Ntani G, Javaid K, Cooper P, Moon R, et al. Vitamin D supplementation in pregnancy: a systematic review. Health Technol Assess 2014;18:1-190.

11. Moher D, Liberati A, Tetzlaff J, Altman DG. Preferred reporting items for systematic reviews and meta-analyses: the PRISMA statement. Int J Surg 2010; 8:336-41.

12. Higgins JPT, Green S, editors. Cochrane Handbook for Systematic Reviews of Interventions. Version 5.1.0. Available at: http://www. cochrane-handbook. org. Accessed July 23, 2014.

13. Mantel N, Haenszel W. Statistical aspects of the analysis of data from retrospective studies of disease. J Natl Cancer Inst 1959;22:719-48.

14. Review Manager (RevMan) [Computer program]. Version 5.1. Copenhagen: The Nordic Cochrane Centre, The Cochrane Collaboration, 2011.

15. Asemi Z, Samimi M, Tabassi Z, Shakeri H, Esmaillzadeh A. Vitamin D supplementation affects serum high-sensitivity C-reactive protein, insulin resistance, and biomarkers of oxidative stress in pregnant women. J Nutr 2013;143:1432-8.

16. Brooke OG, Brown IR, Bone CD, Carter ND, Cleeve HJ, Maxwell JD, et al. Vitamin D supplements in pregnant Asian women: effects on calcium status and fetal growth. Br Med J 1980;280:751-4.

17. Delvin EE, Salle BL, Glorieux FH, Adeleine P, David LS. Vitamin D supplementation during pregnancy: effect on neonatal calcium homeostasis. J Pediatr 1986;109:328-34.

18. Goldring ST, Griffiths CJ, Martineau AR, Robinson S, Yu C, Poulton S, et al. Prenatal vitamin D supplementation and child respiratory health: a randomised controlled trial. PLoS One 2013;8:e66627.

19. Hashemipour S, Ziaee A, Javadi A, Movahed F, Elmizadeh K, Javadi EH, et al. Effect of treatment of vitamin D deficiency and insufficiency during pregnancy on fetal growth indices and maternal weight gain: a randomized clinical trial. Eur J Obstet Gynecol Reprod Biol 2014;172:15-9.

20. Hossain N, Kanani FH, Ramzan S, Kausar R, Ayaz S, Khanani R, et al. Obstetric and neonatal outcomes of maternal vitamin $\mathrm{D}$ supplementation: results of an open label randomized controlled trial of antenatal vitamin D supplementation in Pakistani women. J Clin Endocrinol Metab 2014;99:2448-55.

21. Mallet E, Gügi B, Brunelle P, Hénocq A, Basuyau JP, Lemeur H. Vitamin D supplementation in pregnancy: a controlled trial of two methods. Obstet Gynecol 1986;68:300-4.

22. Marya RK, Rathee S, Manrow M. Effect of calcium and vitamin D supplementation on toxaemia of pregnancy. Gynecol Obstet Invest 1987;24:38-42.

23. Marya RK, Rathee S, Dua V, Sangwan K. Effect of vitamin D supplementation during pregnancy on foetal growth. Indian J Med Res 1988;88:488-92.

24. Roth DE, Al Mahmud A, Raqib R, Akhtar E, Perumal N, Pezzack B, et al. Randomized placebo-controlled trial of high-dose prenatal third-trimester vitamin $D_{3}$ supplementation in Bangladesh: the AViDD trial. Nutr J 2013;12:47.

25. Sabet Z, Ghazi AA, Tohidi M, Oladi B. Vitamin D supplementation in pregnant Iranian women: effects on maternal and neonatal vitamin D and parathyroid hormone status. Acta Endo (Buc) 2012;8:59-66.

26. Yu CK, Sykes L, Sethi M, Teoh TG, Robinson S. Vitamin D deficiency and supplementation during pregnancy. Clin Endocrinol (Oxf) 2009;70:685-90.

27. Kovacs CS. Bone metabolism in the fetus and neonate. Pediatr Nephrol 2014;29:793-803.

28. Villar J, Cheikh Ismail L, Victora CG, Ohuma EO, Bertino E, Altman DG, et al. International standards for newborn weight, length, and head circumference by gestational age and sex: the Newborn Cross-Sectional Study of the INTERGROWTH-21st Project. Lancet 2014;384:857-68.

29. Freemark M. Placental hormones and the control of fetal growth. J Clin Endocrinol Metab 2010;95:2054-7.

30. Shin JS, Choi MY, Longtine MS, Nelson DM. Vitamin D effects on pregnancy and the placenta. Placenta 2010;31:1027-34.

31. Figueras F, Gratacós E. Update on the diagnosis and classification of fetal growth restriction and proposal of a stage-based management protocol. Fetal Diagn Ther 2014;36:86-98.

32. Papageorghiou AT, Ohuma EO, Altman DG, Todros T, Cheikh Ismail L, Lambert A, et al. International standards for fetal growth based on serial ultrasound measurements: the Fetal Growth Longitudinal Study of the INTERGROWTH-21st Project. Lancet 2014;384:869-79.

33. Song SJ, Si S, Liu J, Chen X, Zhou L, Jia G, et al. Vitamin D status in Chinese pregnant women and their newborns in Beijing and their relationships to birth size. Public Health Nutr 2013;16:687-92.

34. Bodnar LM, Rouse DJ, Momirova V, Peaceman AM, Sciscione A, Spong CY, et al. Maternal 25-hydroxyvitamin D and preterm birth in twin gestations. Obstet Gynecol 2013;122:91-8.

35. Thota C, Menon R, Fortunato SJ, Brou L, Lee JE, Al-Hendy A. 1,25Dihydroxyvitamin $\mathrm{D}$ deficiency is associated with preterm birth in African American and Caucasian women. Reprod Sci 2014;21:244-50.

36. Baker AM, Haeri S, Camargo CA Jr, Stuebe AM, Boggess KA. A nested casecontrol study of first-trimester maternal vitamin D status and risk for spontaneous preterm birth. Am J Perinatol 2011;28:667-72.

37. Thorp JM, Camargo CA, McGee PL, Harper M, Klebanoff MA, Sorokin Y, et al. Vitamin D status and recurrent preterm birth: a nested case-control study in high-risk women. BJOG 2012;119:1617-23.

38. Liong S, Di Quinzio MK, Fleming G, Permezel M, Georgiou HM. Is vitamin D binding protein a novel predictor of labour? PLoS One 2013;8:e76490.

39. Bodnar LM, Simhan HN, Catov JM, Roberts JM, Platt RW, Diesel JC, et al. Maternal vitamin D status and the risk of mild and severe preeclampsia. Epidemiology 2014;25:207-14.

40. Bomba-Opon DA, Brawura-Biskupski-Samaha R, Kozlowski S, Kosinski P, Bartoszewicz Z, Bednarczuk T, et al. First trimester maternal serum vitamin D and markers of preeclampsia. J Matern Fetal Neonatal Med 2014;27:1078-9.

41. Ma R, Gu Y, Zhao S, Sun J, Groome LJ, Wang Y. Expressions of vitamin D metabolic components VDBP, CYP2R1, CYP27B1, CYP24A1, and VDR in placentas from normal and preeclamptic pregnancies. Am J Physiol Endocrinol Metab 2012;303:E928-35.

42. Hofmeyr GJ, Belizán JM, von Dadelszen P, Calcium and Pre-eclampsia (CAP) Study Group. Low-dose calcium supplementation for preventing preeclampsia: a systematic review and commentary. BJOG 2014;121:951-7.

43. Hofmeyr GJ, Lawrie TA, Atallah AN, Duley L, Torloni MR. Calcium supplementation during pregnancy for preventing hypertensive disorders and related problems. Cochrane Database Syst Rev 2014;6:CD001059. 
44. Burris HH, Rifas-Shiman SL, Kleinman K, Litonjua AA, Huh SY, RichEdwards JW, et al. Vitamin D deficiency in pregnancy and gestational diabetes mellitus. Am J Obstet Gynecol 2012;207:182.e1-8.

45. Poel YH, Hummel P, Lips P, Stam F, van der Ploeg T, Simsek S. Vitamin D and gestational diabetes: a systematic review and meta-analysis. Eur J Intern Med 2012;23:465-9.

46. Knabl J, Hüttenbrenner R, Hutter S, Günthner-Biller M, Riedel C, Hiden U, et al. Gestational diabetes mellitus upregulates vitamin D receptor in extravillous trophoblasts and fetoplacental endothelial cells. Reprod Sci 2015;22: 358-66.

47. Wamberg L, Christiansen T, Paulsen SK, Fisker S, Rask P, Rejnmark L, et al. Expression of vitamin D-metabolizing enzymes in human adipose tissuethe effect of obesity and diet-induced weight loss. Int J Obes (Lond) 2013; 37:651-7.

48. Karlsson T, Osmancevic A, Jansson N, Hulthén L, Holmäng A, Larsson I. Increased vitamin D-binding protein and decreased free 25-OHD in obese women of reproductive age. Eur J Nutr 2014;53:259-67.

49. Merewood A, Mehta SD, Chen TC, Bauchner H, Holick MF. Association between vitamin D deficiency and primary cesarean section. J Clin Endocrinol Metab 2009;94:940-5.

50. Scholl TO, Chen X, Stein P. Maternal vitamin D status and delivery by cesarean. Nutrients 2012;4:319-30.

51. Savvidou MD, Makgoba M, Castro PT, Akolekar R, Nicolaides KH. Firsttrimester maternal serum vitamin D and mode of delivery. Br J Nutr 2012; 108:1972-5.

52. Wootton AM. Improving the measurement of 25-hydroxyvitamin D. Clin Biochem Rev 2005;26:33-6.

53. Vassalle C, Pérez-López FR. The importance of some analytical aspects and confounding factors in relation to clinical interpretation of results. In: Meer C, Smits H, editors. Vitamin D: Daily requirements, dietary sources and symptoms of deficiency. New York: Nova Biomedicals; 2013:91-114.

54. Black RE, Allen LH, Bhutta ZA, Caulfield LE, de Onis M, Ezzati M, et al. Maternal and child undernutrition: global and regional exposures and health consequences. Lancet 2008;371:243-60.
55. Josefson JL, Feinglass J, Rademaker AW, Metzger BE, Zeiss DM, Price HE, et al. Maternal obesity and vitamin D sufficiency are associated with cord blood vitamin D insufficiency. J Clin Endocrinol Metab 2013:98:114-9.

56. Young BE, McNanley TJ, Cooper EM, McIntyre AW, Witter F, Harris ZL, et al. Maternal vitamin D status and calcium intake interact to affect fetal skeletal growth in utero in pregnant adolescents. Am J Clin Nutr 2012;95:1103-12.

57. Lieben L, Stockmans I, Moermans K, Carmeliet G. Maternal hypervitaminosis $D$ reduces fetal bone mass and mineral acquisition and leads to neonatal lethality. Bone 2013;57:123-31.

58. Burris HH, Rifas-Shiman SL, Huh SY, Kleinman K, Litonjua AA, Oken E, et al. Vitamin D status and hypertensive disorders in pregnancy. Ann Epidemiol 2014;24:399-403.e1.

59. Ross AC, Manson JE, Abrams SA, Aloia JF, Brannon PM, Clinton SK, et al. The 2011 report on dietary reference intakes for calcium and vitamin D from the Institute of Medicine: what clinicians need to know. J Clin Endocrinol Metab 2011;96:53-8.

60. Ross AC, Taylor CL, Yaktine AL, Del Valle HB, editors. Dietary reference intakes for calcium and vitamin D. Washington, DC: National Academies Press; 2011.

61. Holick MF, Binkley NC, Bischoff-Ferrari HA, Gordon CM, Hanley DA, Heaney RP, et al. Evaluation, treatment, and prevention of vitamin D deficiency: an Endocrine Society clinical practice guideline. J Clin Endocrinol Metab 2011;96:1911-30.

62. ACOG Committee on Obstetric Practice. Committee Opinion Number 495: Vitamin D: screening and supplementation during pregnancy. Obstet Gynecol 2011;118:197-8.

63. Pérez-López FR. Low maternal vitamin D status during pregnancy requires appropriate therapeutic intervention. Int J Gynaecol Obstet 2012;116:4-5.

64. Blumfield ML, Hure AJ, Macdonald-Wicks L, Smith R, Collins CE. A systematic review and meta-analysis of micronutrient intakes during pregnancy in developed countries. Nutr Rev 2013;71:118-32. 


\section{SUPPLEMENTAL TABLE 1}

Search strategies used for PubMed to identify randomized clinical trials to be included in a systematic review and meta-analysis to evaluate the effect of vitamin D supplementation during pregnancy on maternal and neonatal outcomes.

Preeclampsia

\#1: "Vitamin D"[Mesh] OR "Ergocalciferols"[Mesh] OR "Vitamin D Deficiency"[Mesh] OR "Cholecalciferol"[Mesh] OR "Calcifediol"[Mesh] OR "Ergocalciferol" OR "Vitamin D Supplementation" OR "25-hydroxy-vitamin D"

\#2: "Pre-Eclampsia"[Mesh] OR "Preeclampsia" OR "Pre eclampsia" OR "Maternal Hypertension" OR "Hypertensive Disorders of Pregnancy" \#3: \#1 AND \#2

Filters: clinical trial, randomized clinical trial

Gestational diabetes

\#1: "Vitamin D"[Mesh] OR "Ergocalciferols"[Mesh] OR "Vitamin D Deficiency"[Mesh] OR "Cholecalciferol"[Mesh] OR "Calcifediol"[Mesh] OR "Ergocalciferol" OR "Vitamin D Supplementation" OR "25-hydroxy-vitamin D"

\#2: "Diabetes, Gestational"[Mesh] OR "Gestational Diabetes" OR "Gestational Diabetes Mellitus" OR "Diabetes Pregnancy Induced"

\#3: \#1 AND \#2

Filters: clinical trial, randomized clinical trial

VItamin D status at term

\#1: "Vitamin D"[Mesh] OR "Ergocalciferols"[Mesh] OR "Vitamin D Deficiency"[Mesh] OR "Cholecalciferol"[Mesh] OR "Calcifediol"[Mesh] OR "Ergocalciferol" OR "Vitamin D Supplementation" OR "25-hydroxy-vitamin D" OR "Vitamin D Level" OR "Vitamin D Status" OR "25(OH) D levels"

\#2: "Pregnancy" OR "End of Pregnancy" OR "At Term Pregnancy"

\#3: \#1 AND \#2

Filters: clinical trial, randomized clinical trial

Fetal growth retardation

\#1: "Vitamin D"[Mesh] OR "Ergocalciferols"[Mesh] OR "Vitamin D Deficiency"[Mesh] OR "Cholecalciferol"[Mesh] OR "Calcifediol"[Mesh] OR "Ergocalciferol" OR "Vitamin D Supplementation" OR "25-hydroxy-vitamin D"

\#2: "Fetal Growth Retardation"[Mesh] OR "Intrauterine Growth Retardation" OR "Intrauterine Growth Restriction" OR "Fetal Growth Restriction"

\#3: \#1 AND \#2

Filters: clinical trial, randomized clinical trial

Low and very low birth weight

\#1: "Vitamin D"[Mesh] OR "Ergocalciferols"[Mesh] OR "Vitamin D Deficiency"[Mesh] OR "Cholecalciferol"[Mesh] OR "Calcifediol"[Mesh] OR "Ergocalciferol" OR "Vitamin D Supplementation" OR "25-hydroxy-vitamin D"

\#2: "Infant, Extremely Low Birth Weight"[Mesh] OR "Infant, Very Low Birth Weight"[Mesh] OR "Infant, Low Birth Weight"[Mesh] OR "Infant, Small for Gestational Age"[Mesh] OR "Low Birth Weight"

\#3: \#1 AND \#2

Filters: clinical trial, randomized clinical trial

Premature and very premature birth

\#1: "Vitamin D"[Mesh] OR "Ergocalciferols"[Mesh] OR "Vitamin D Deficiency"[Mesh] OR "Cholecalciferol"[Mesh] OR "Calcifediol"[Mesh] OR "Ergocalciferol" OR "Vitamin D Supplementation" OR "25-hydroxy-vitamin D"

\#2: "Premature Birth"[Mesh] OR "Preterm Birth" OR "Premature Newborn" OR "Preterm Labor" OR "Very Premature Birth"

\#3: \#1 AND \#2

Filters: clinical trial, randomized clinical trial

Birth weight

\#1: "Vitamin D"[Mesh] OR "Ergocalciferols"[Mesh] OR "Vitamin D Deficiency"[Mesh] OR "Cholecalciferol"[Mesh] OR "Calcifediol"[Mesh] OR "Ergocalciferol" OR "Vitamin D Supplementation" OR "25-hydroxy-vitamin D"

\#2: "Birth weight" [Mesh] or "birth weight"

\#3: \#1 AND \#2

Filters: clinical trial, randomized clinical trial

Cesarean section

\#1: "Vitamin D"[Mesh] OR "Ergocalciferols"[Mesh] OR "Vitamin D Deficiency"[Mesh] OR "Cholecalciferol"[Mesh] OR "Calcifediol"[Mesh] OR "Ergocalciferol" OR "Vitamin D Supplementation" OR "25-hydroxy-vitamin D"

\#2: "Cesarean Section"[Mesh] OR "Cesarean Delivery" OR "Born by Cesarean"

\#3: \#1 AND \#2

Filters: clinical trial, randomized clinical trial

Maternal mortality

\#1: "Vitamin D"[Mesh] OR "Ergocalciferols"[Mesh] OR "Vitamin D Deficiency"[Mesh] OR "Cholecalciferol"[Mesh] OR "Calcifediol"[Mesh] OR "Ergocalciferol" OR "Vitamin D Supplementation" OR "25-hydroxy-vitamin D"

\#2: "Maternal Mortality"[Mesh] OR "Maternal Mortalities" OR "Maternal Deaths"

\#3: \#1 AND \#2

Filters: clinical trial, randomized clinical trial

Birth length

\#1: "Vitamin D"[Mesh] OR "Ergocalciferols"[Mesh] OR "Vitamin D Deficiency"[Mesh] OR "Cholecalciferol"[Mesh] OR "Calcifediol"[Mesh] OR "Ergocalciferol" OR "Vitamin D Supplementation" OR "25-hydroxy-vitamin D"

\#2: "Birth Length" OR "Size at Birth" OR "Stature at Birth"

\#3: \#1 AND \#2

Filters: clinical trial, randomized clinical trial

Apgar score $<7$ at 5 min

\#1: "Vitamin D"[Mesh] OR "Ergocalciferols"[Mesh] OR "Vitamin D Deficiency"[Mesh] OR "Cholecalciferol"[Mesh] OR "Calcifediol"[Mesh] OR "Ergocalciferol" OR "Vitamin D Supplementation" OR "25-hydroxy-vitamin D"

\#2: "Apgar Score"[Mesh] OR "Apgar test"

Pérez-López. Vitamin D supplementation and pregnancy. Fertil Steril 2015. 


\section{SUPPLEMENTAL TABLE 1}

\section{Continued.}

\#3: \#1 AND \#2

Filters: clinical trial, randomized clinical trial

Still birth

\#1: "Vitamin D"[Mesh] OR "Ergocalciferols"[Mesh] OR "Vitamin D Deficiency"[Mesh] OR "Cholecalciferol"[Mesh] OR "Calcifediol"[Mesh] OR "Ergocalciferol" OR "Vitamin D Supplementation" OR "25-hydroxy-vitamin D"

\#2: "Stillbirth"[Mesh]) OR "Fetal Death"[Mesh]

\#3: \#1 AND \#2

Filters: clinical trial, randomized clinical trial

Neonatal infection

\#1: "Vitamin D"[Mesh] OR "Ergocalciferols"[Mesh] OR "Vitamin D Deficiency"[Mesh] OR "Cholecalciferol"[Mesh] OR "Calcifediol"[Mesh] OR "Ergocalciferol" OR "Vitamin D Supplementation" OR "25-hydroxy-vitamin D"

\#2: "Neonatal Infection" OR "Neonatal Infectious Diseases"

\#3: \#1 AND \#2

Filters: clinical trial, randomized clinical trial

Neonatal mortality

\#1: "Vitamin D"[Mesh] OR "Ergocalciferols"[Mesh] OR "Vitamin D Deficiency"[Mesh] OR "Cholecalciferol"[Mesh] OR "Calcifediol"[Mesh] OR "Ergocalciferol" OR "Vitamin D Supplementation" OR "25-hydroxy-vitamin D"

\#2: "Infant Mortality"[Mesh] OR "Neonatal Mortality" OR "Neonatal Mortalities"

\#3: \#1 AND \#2

Filters: clinical trial, randomized clinical trial

Pérez-López. Vitamin D supplementation and pregnancy. Fertil Steril 2015. 


\section{SUPPLEMENTAL TABLE 2}

Comparison of recently published meta-analyses and our study of randomized clinical trials in a systematic review and meta-analysis to evaluate the effect of vitamin D supplementation during pregnancy on maternal and neonatal outcomes.

\section{Study, first author (reference \# in text)}

\section{Characteristic}

Year of publication

Type of review

Type of studies included

Primary objectives

Type of participants

Type of intervention

Exclusion criteria

No. of studies included in quantitative synthesis

Sample size in quantitative synthesis

Quality assessment

GRADE assessment review

RCTs and quasicalcium or other gestational or of fetuses

Vitamin D long as the treated similarly studies

\section{6}

32-502
Thorne-Lyman (7)

2012

Cochrane systematic randomized trials

To examine whether supplements with vitamin $\mathrm{D}$ alone or in combination with vitamins and minerals given to women during pregnancy can safely improve maternal and neonatal outcomes

Pregnant women of any chronologic age, parity (no. of births), and no.

supplementation during pregnancy irrespective of dose, duration, or time of commencement; trials testing vitamin $\mathrm{D}$ alone or in combination with other micronutrients as intervention and the control group were

Crossover trials or any other observational

As substantial statistical heterogeneity was detected, randomeffects meta-analysis to produce an overall summary of an average
Systematic review and meta-analysis

RCTs and observational studies

To assess the effect of vitamin D supplementation, intake or $25(\mathrm{OH}) \mathrm{D}$ status during pregnancy on perinatal and infant health outcomes

Pregnant women

Vitamin D from supplements or diet

(1) nonhuman studies; (2) studies not in English, French, or Spanish; (3) reviews, case reports, and commentaries; (4) topics unrelated to the review; (5) studies that could not isolate the effects of vitamin D supplementation or intake; and (6) crosssectional and nonprospective case-

5 trials and 2 observational studies

126-228

Modified GRADE tool

Inverse variance weights were used to generate pooled effect estimates with a fixedeffects model in absence of significant heterogeneity. In the control studies
Harvey (10)

\section{4}

Systematic review

RCTs and observational studies

To investigate whether maternal

supplementation with vitamin D in pregnancy

leads to an

improvement in

maternal and neonatal outcomes

Pregnant women or pregnant women and their offspring

Assessment of vitamin D status (dietary intake, sunlight exposure, circulating 25(OH)D concentration) or supplementation of participants with vitamin D or food containing vitamin $\mathrm{D}$ (e.g., oily fish)

Studies were excluded if they were not written in English, were nonhuman studies, did not measure maternal vitamin D status in or immediately after pregnancy or supplement participants with vitamin D in pregnancy, or where an outcome of interest was not measured. 19

NR

Refined questionnaire based on Center for Reviews and Dissemination guidelines

If no significant heterogeneity was noted, fixed-effect model analysis using the Mantel-Haenszel method; otherwise, results of the random-

\section{Current study}

2015

Systematic review and RCTs meta-analysis

To evaluate the efficacy of vitamin D

supplementation during pregnancy on obstetric and neonata outcomes in RCTs

Pregnant women of any gestational or chronologic age and parity, without previous disease history

Interventions of interest were: vitamin D alone vs. no treatment/ placebo; vitamin D + calcium vs. no treatment/placebo; and vitamin D + calcium vs. calcium. Controls of interest were active controls, usual treatment without active control, and placebo

(1) no relevant control group; (2) data were not available or could not extracted for the study groups; and (3) studies that included a combination therapy of vitamin D + calcium + other vitamins and minerals

Cochrane Collaboration Handbook risk-of-bias assessment tool

Fixed-effects models and the Mantel-Haenszel method 
SUPPLEMENTAL TABLE 2

\section{Continued.}

\section{Characteristic}

Primary outcomes

Secondary outcomes

Outcome association measure with $95 \% \mathrm{Cl}$

\section{De Regil (9)}

treatment effect across trials was used.

Maternal (preeclampsia, GDM, vitamin D status at term); infant (preterm birth, LBW)

Maternal (impaired glucose tolerance, cesarean section, gestational HTN, side effect, maternal death); infant (birth length and weight, head circumference, stillbirth, neonatal death, ICU admission, Apgar score, neonatal infection, very preterm birth)

Preeclampsia (RR 0.67, 95\% Cl 0.33-1.35); LBW (RR $0.48,95 \% \mathrm{CI}$ 0.23-1.01)

\section{Vitamin D}

supplementation in a single or continued dose during pregnancy increases serum vitamin D concentrations as measured by 25hydroxyvitamin $\mathrm{D}$ at term. The clinical significance of this finding and the potential use of this intervention as a part of routine antenatal care are yet to be determined, as the number of high-quality trials and outcomes reported is too limited to draw conclusions on its usefulness and safety.

\section{Study, first author (reference \# in text)}

\section{Thorne-Lyman (7)}

presence of significant heterogeneity, random-effects model was used. growth restriction, preterm birth; neonatal growth/ morbidity/mortality; infant growth/ morbidity/mortality maternal morbidity and mortality NR
LBW due to intrauterine

effects model analysis using the DerSimonian-Laird method were used

Maternal osteomalacia, neonatal

hypocalcemia, rickets, and reduced bone mass

Maternal quality of life, neonatal body composition, and later offspring health outcomes (including asthma, diabetes mellitus, and immune disease)

Protective effects of LBW (RR 0.40, 95\% Cl 0.23-0.71) and nonsignificant but of daily supplementation on SGA (RR 0.67, 95\% Cl 0.40-1.11]); no effect on preterm delivery (<37 wk) was evident (RR $0.77,95 \% \mathrm{Cl}$ 0.35-1.66)

Little evidence from trials exists to evaluate the effect of vitamin D supplementation during pregnancy on maternal, perinatal, or infant health outcomes. Based on both trials and observational studies, we recommend that future research explore SGA, preterm delivery, preeclampsia, and maternal and childhood infections, as outcomes of interest.

\section{Harvey (10)} supplementation on suggestive effects
Maternal 25(OH)D was associated with birth weight in metaanalysis of 3 observational studies using log-transformed 25(OH)D

concentrations after adjustment for potential confounding factors (pooled regression coefficient $5.63 \mathrm{~g} / 10 \%$ change maternal 25(OH)D, 95\% Cl 1.11-10.16 g)

The evidence base is currently insufficient to support definite clinical recommendations regarding vitamin $\mathrm{D}$ supplementation in pregnancy. Although there is modest evidence to support a relationship between maternal $25(\mathrm{OH}) \mathrm{D}$ status and offspring birth weight, bone mass and serum calcium concentrations, these findings were limited by their observational nature (birth weight, bone mass) or risk of bias and low quality

(calcium concentrations).
Maternal (preeclampsia, gestational diabetes, change of vitamin $D$ levels at term); neonatal (SGA, LBW, preterm birth, birth weight)

Maternal (cesarean section and maternal mortality); neonatal (birth length, Apgar score, stillbirth, neonatal infection, very low weight $(<1,500 \mathrm{~g})$, very low preterm birth (<34 wk gestation), and neonatal mortality

Preeclampsia (RR 0.88, 95\% Cl 0.51-1.52); gestational diabetes (RR 1.05, 95\% Cl $0.60-1.84) ;$ SGA (RR $0.78,95 \%$ Cl 0.501.21); low birth weight (RR $0.72,95 \% \mathrm{Cl}$ $0.44-1.16) ;$ and preterm birth (RR 1.26, 95\% Cl 0.60-2.63)

According to existing studies, vitamin D supplementation during pregnancy is not associated with better maternal and neonatal outcomes. Larger RCTs with better designs that evaluate clinically relevant outcomes are necessary to reach a definitive conclusion. 